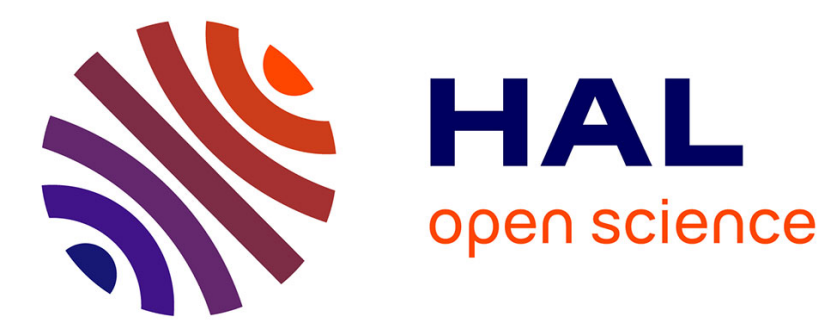

\title{
Combining sources of evidence with reliability and importance for decision making
}

Lianmeng Jiao, Quan Pan, Yan Liang, Xiaoxue Feng, Feng Yang

\section{To cite this version:}

Lianmeng Jiao, Quan Pan, Yan Liang, Xiaoxue Feng, Feng Yang. Combining sources of evidence with reliability and importance for decision making. Central European Journal of Operations Research, 2016, 24 (1), pp.87-106. 10.1007/s10100-013-0334-3 . hal-01080973

\section{HAL Id: hal-01080973 https://hal.science/hal-01080973}

Submitted on 9 Jan 2015

HAL is a multi-disciplinary open access archive for the deposit and dissemination of scientific research documents, whether they are published or not. The documents may come from teaching and research institutions in France or abroad, or from public or private research centers.
L'archive ouverte pluridisciplinaire HAL, est destinée au dépôt et à la diffusion de documents scientifiques de niveau recherche, publiés ou non, émanant des établissements d'enseignement et de recherche français ou étrangers, des laboratoires publics ou privés. 


\title{
Combining sources of evidence with reliability and importance for decision making
}

\author{
Lianmeng Jiao • Quan Pan • Yan Liang • \\ Xiaoxue Feng - Feng Yang
}

Received: / Accepted:

\begin{abstract}
The combination of sources of evidence with reliability has been widely studied within the framework of Dempster-Shafer theory (DST), which has been employed as a major method for integrating multiple sources of evidence with uncertainty. By the fact that sources of evidence may also be different in importance, for example in multi-attribute decision making (MADM), we propose the importance discounting and combination method within the framework of DST to combine sources of evidence with importance, which is composed of an importance discounting operation and an extended Dempster's rule of combination. Three evidence combination axioms are proposed and explored to uncover the differences between reliability and importance in evidence reasoning. Furthermore, a general scheme is proposed for combination of sources of evidence with both reliability and importance. An example of car performance evaluation is studied to show the efficiency of the new general scheme.
\end{abstract}

Keywords Decision making - Evidence theory · Importance discounting operation · Extended Dempster's rule of combination

\section{Introduction}

In many real-life fusion problems, one has to deal with different sources of information arising from human reports, artificial intelligent systems or physical sensors, which are usually imprecise, uncertain, or incomplete. It's essential to combine all

This work is partially supported by China Natural Science Foundation (No. 61135001, 61075029) and the Doctorate Foundation of Northwestern Polytechnical University (No. CX201319).

L. Jiao · Q. Pan · Y. Liang · X. Feng · F. Yang

School of Automation, Northwestern Polytechnical University,

Xi'an, 710072, P. R. China.

Tel.: +86-29-88431307

Fax: +86-29-88431306

E-mail: jiaolianmeng@mail.nwpu.edu.cn; liangyan@nwpu.edu.cn 
the information in a way that one has a better understanding and assessment of the complex problem under consideration for decision-making support. DempsterShafer theory (DST) (Dempster 1967; Shafer 1976) or evidence theory, as a general extension of Bayesian theory, is a common and efficient method employed to solve the high-level fusion problems in expert reasoning (Shafer 1987; Yang et al 2006; Ghasemi et al 2013), pattern recognition (Denœux 1995; Denœux and Masson 2012; Tabassian et al 2012), data mining (Anand et al 1996; Scotney and McClean 2003; Srinivasan et al 2005) and decision making (Beynon et al 2001; Davis and Hall 2003; Xu 2012).

Within the framework of DST, the reliability of different sources is usually considered in the evidence aggregation process due to the sources' limitation to provide totally accurate information (Hégarat-Mascle et al 1998; Milisavljević and Bloch 2003). As for the combination problem of sources of evidence with reliability, usually the classical Shafer's discounting operation (Shafer 1976) is applied before the classical Dempster's rule of combination (Dempster 1967). However, in some decision making problems, especially for MADM, different attributes should be weighted in order to get more reasonable fusion results (Hwang and Yoon 1981). While in some previous works (Yang and Sen 1994; Beynon et al 2000; Beynon $2005 \mathrm{~b})$, the weights of attributes are utilized according to Shafer's discounting operation, which makes no difference with the treatment of reliability. This kind of management fails to follow the common sense rules (Yang and Xu 2002) that a rational aggregation process needs to satisfy. Until recently, the importance notion of one source of evidence is proposed to describe the weight of the corresponding attribute (Tacnet et al 2009). The authors believe that the reliability and importance characterize the properties of a source of evidence in different aspects and thus should be distinguished in the fusion process.

More recently, a method for combining sources of evidence with importance has been established (Smarandache et al 2010) within the framework of DezertSmarandache theory (DSmT), which extends DST by refuting the exclusivity constraint on the frame of discernment. However, it's explored that the proposed method has some problems as follows. Firstly, the combination rule developed in DSmT to integrate the discounted sources of evidence is not associative and hence will become quite computationally demanding as the number of sources of evidence increases (Smarandache and Dezert 2006). More seriously, the utilized discounting operation transfers all the remaining discounted mass to the empty set. We believe it is theoretically irrational as the empty set is already used to characterize the conflicting information in their operation and the remaining discounted mass essentially represents the indecisiveness rather than the conflicting information.

As discussed above, although distinct notion is presented for importance from reliability, there is still no theoretically sound method to address the evidence reasoning problem with importance in DST framework and the essential differences between reliability and importance is also unclear. Motivated by the above consideration, in this paper, an importance discounting operation is defined that commits the remaining discounted mass not to the empty set but a new set to characterize the indecisiveness that needs to be jointly assigned with other new importance discounted sources. Then, an extended Dempster's rule of combination is developed to combine the importance discounted sources of evidence in DST framework, which is both commutative and associative and thus has a relatively low computing burden. Three evidence combination axioms (independence, 
consensus and completeness) are proposed and explored, which reveal essential differences between reliability and importance and illustrate the proposed method in more meaningful ways. Furthermore, a reliability-importance discounting and combination method is developed, which provides a general scheme for combination of sources of evidence. Our new general scheme is illustrated via a car performance evaluation example.

This paper is organized as follows. In Sect. 2, we firstly give a brief reminder of the basic notions in DST, and then the reliability discounting and combination method is revisited and analyzed. In Sect. 3, we propose the method for taking into account the importance of sources of evidence in the combination process and then three evidence combination axioms are proposed and explored in Sect. 4. Sect. 5 develops a general scheme for combination of sources of evidence with reliability and importance, and then an example of car performance evaluation is examined in Sect. 6. Finally, Sect. 7 presents some concluding remarks.

\section{Reliability discounting and combination}

The combination of sources of evidence with reliability has been widely studied within the framework of DST. To facilitate later discussion, in this section, we first briefly review the basic concepts of DST, and then present the concept of reliability and its acquisition method. Finally, the classical reliability discounting and combination method is revisited and analyzed.

\subsection{Basic concepts in DST}

To begin with, let us introduce some basic concepts in DST (Shafer 1976). In DST, a problem domain is represented by a finite set $\Theta=\left\{\theta_{1}, \theta_{2}, \cdots, \theta_{n}\right\}$ of mutually exclusive and exhaustive hypotheses called the frame of discernment. A basic belief assignment (BBA) expressing the belief committed to the elements of $2^{\Theta}$ by a given source of evidence is a mapping function $\mathrm{m}(\cdot): 2^{\Theta} \rightarrow[0,1]$, such that

$$
\mathrm{m}(\emptyset)=0 \text { and } \sum_{A \in 2^{\Theta}} \mathrm{m}(A)=1
$$

where, $2^{\Theta}$ denotes the power set of $\Theta$, consisting all the $2^{n}$ subsets of $\Theta$. Elements $A \in 2^{\Theta}$ having $\mathrm{m}(A)>0$ are called focal elements of the $\operatorname{BBA} \mathrm{m}(\cdot) . \operatorname{ABBA} \mathrm{m}(A)$ measures the degree of belief exactly assigned to a proposition $A$ and represents how strongly the proposition is supported by evidence. The belief assigned to all the subsets of $2^{\Theta}$ is summed to unity and there is no belief left to the empty set. The belief assigned to $\Theta$, or $\mathrm{m}(\Theta)$, is referred to as the degree of global ignorance.

Shafer also defines the belief and plausibility functions of $A \in 2^{\Theta}$ as follows

$$
\operatorname{Bel}(A)=\sum_{B \subseteq A} \mathrm{~m}(B) \text { and } \operatorname{Pl}(A)=\sum_{B \cap A \neq \emptyset} \mathrm{m}(B) .
$$

$\operatorname{Bel}(A)$ represents the exact support to $A$ and its subsets, and $\operatorname{Pl}(A)$ represents all the possible support to $A$ and its subsets. The interval $[\operatorname{Bel}(A), \mathrm{Pl}(A)]$ can be seen as the lower and upper bounds of support to $A$. The belief functions $\mathrm{m}(\cdot), \operatorname{Bel}(\cdot)$ and $\mathrm{Pl}(\cdot)$ are in one-to-one correspondence. 
For decision-making support, the pignistic probability $\operatorname{BetP}(A)$ (Smets 2005) is commonly used to approximate the unknown probability in $[\operatorname{Bel}(A), \operatorname{Pl}(A)]$, given by

$$
\operatorname{BetP}(A)=\sum_{\substack{B \subseteq \Theta \\ A \cap B \neq \emptyset}} \frac{|A \cap B|}{|B|} \mathrm{m}(B),
$$

where, $|X|$ stands for the cardinality of the element $X$.

\subsection{Reliability and its acquisition}

As in (Smarandache et al 2010), the definition of reliability for a source of evidence in the context of DST is given as follows.

Definition 1 (reliability of a source of evidence) The reliability, as an objective property of a source of evidence, represents its capability to provide correct measure or assessment of the considered problem.

Remark 1 The reliability of a source of evidence can be characterized by a reliability factor $\alpha \in[0,1]$, where $\alpha=1$ and $\alpha=0$ represents that the source is fully reliable and totally unreliable, respectively. Besides, according to this definition, the reliability of one source of evidence is determined only by itself and the reliability factors of different sources of evidence involved in the fusion process is independent with each other.

The reliability of a source of evidence does exit in many fusion problems and generally can be acquired in the following two ways:

- The reliability of a source of evidence is prior-known or can be determined from some available properties (Haenni and Hartmann 2006). For example, it may be constructed from the known measurement accuracy of the given sensor. Sometimes, this reliability would be context-dependent (Mercier et al 2008). For example, if a sensor more possibly does not perform well under bad weather conditions, then the corresponding reliability of information from that sensor will be expected to become smaller.

- The reliability of a source of evidence is approximately estimated from the dissimilarity with the other sources of evidence (Deng et al 2004; Liu et al 2011). Generally, for any a source of evidence, its larger dissimilarity with other sources measures the lower reliability.

\subsection{Shafer's discounting operation and Dempster's rule of combination}

The reliability of a source of evidence is generally considered according to Shafer's discounting method (Shafer 1976). Mathematically, Shafer's discounting operation (detonated as $\otimes$ ) for taking into account the reliability factor $\alpha \in[0,1]$ of a given source with a BBA $\mathrm{m}(\cdot)$ and a frame $\Theta$ is defined by

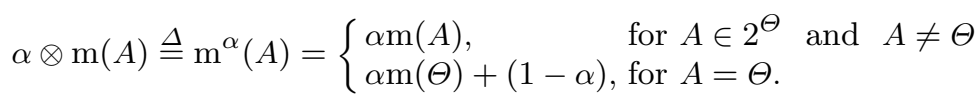


As shown in Eq.(3), Shafer's discounting method multiplies the masses of focal elements by the reliability factor $\alpha$, and transfers all the remaining discounted mass $1-\alpha$ to the global ignorance $\Theta$. By the fact that $\alpha \mathrm{m}(\Theta)+(1-\alpha)>\alpha \mathrm{m}(\Theta)+$ $(1-\alpha) \mathrm{m}(\Theta)=\mathrm{m}(\Theta)$ in case of $\mathrm{m}(\Theta)<1$ and $\alpha<1$, such discounting operation almost always increases the global ignorance.

After the reliability discounting operation, Dempster's rule of combination (the orthogonal sum operation $\oplus$ ) is usually used to combine two discounted pieces of evidence on the same frame $\Theta$ represented by two BBAs $m_{1}^{\alpha_{1}}(\cdot)$ and $m_{2}^{\alpha_{2}}(\cdot)$ to generate a new BBA, denoted by $\mathrm{m}_{12}^{\mathrm{D}}(\cdot)$

$\mathrm{m}_{1}^{\alpha_{1}} \oplus \mathrm{m}_{2}^{\alpha_{2}}(A) \triangleq \mathrm{m}_{12}^{\mathrm{D}}(A)= \begin{cases}0, & \text { for } A=\emptyset \\ \frac{\sum_{B, C \in 2}{ }_{; B \cap C=A}}{1-\sum_{B, C \in 2} \sum_{; B \cap \cap C=\emptyset}^{\alpha_{1}}(B) \mathrm{m}_{2}^{\alpha_{2}}(C)} \mathrm{m}_{1}^{\alpha_{1}}(B) \mathrm{m}_{2}^{\alpha_{2}(C)} & \text { for } A \in 2^{\Theta} \text { and } A \neq \emptyset .\end{cases}$

As described in (Shafer 1976), Dempster's rule of combination is both commutative and associative.

\section{Importance discounting and combination}

The importance of a source plays significant role in the process of MADM and it is quite different from the reliability of that source. Specifically, the importance usually reflect the decision maker's subjective preference to this kind of source while the reliability is a objective property representing the source's capability to provide the correct measure or assessment and thus they should be addressed in different ways. This section aims to study the fusion problem of sources of evidence with importance in DST framework.

\subsection{Importance and its acquisition}

Distinct from the concept of reliability, as in (Smarandache et al 2010), the importance of a source of evidence in the context of DST can be defined as follows.

Definition 2 (importance of a source of evidence) The importance, as a subjective property of a source of evidence, represents the weight of that source which reflects the decision maker's subjective preference in the fusion process.

Remark 2 Different from the reliability defined in Sect. 2, the defined importance belongs to a relative notion. In other words, the importance of one source is meaningless without comparing with other sources. It can be characterized by an importance factor, denoted $\beta \in[0,1]$. The decision maker will take $\beta=1$ when he/she wants to grant the maximum importance to the source in the fusion process, and will take $\beta=0$ if no importance at all is granted to this source.

The importance of a source of evidence (or the relative weight of the corresponding attribute) can be determined in the following two ways: 
- In most cases, weights are selected based on preference information of attributes given by the fusion system designers (FSDs) or the decision makers (DMs). The commonly-used methods include direct rating method (Roberts and Goodwin 2002), ranking ordering method (Barron and Barrett 1996), and analytic hierarchy process (AHP) method (Ishizaka and Lusti 2006; Dijkstra 2013), which reflect the subjective intuition or the knowledge of FSDs or DMs.

- Sometimes the relative weight of the attribute will be determined from some available properties with the respect to the importance of the attribute, for example, the entropy of the decision matrix (Xu 2004).

\subsection{Importance discounting operation}

In this subsection, we propose a solution to consider the importance of sources of evidence in DST framework. Our definition of importance discounting operation draws inspiration from the weight addressing technique in ER algorithm for MADM (Yang and Xu 2002; Xu et al 2006; Yang and Xu 2011). We define this operation in the viewpoint of "importance" as the counterpart of Shafer's discounting operation for reliability of sources of evidence. It commits the remaining discounted mass to a new set $\Omega$ rather than the global ignorance set $\Theta$ as done by Shafer in reliability discounting presented in Sect. 2.

Definition 3 (importance discounting operation) The importance discounting operation (detonated as $\odot$ ) of a source of evidence having the importance factor $\beta \in[0,1]$ and associated BBA $\mathrm{m}(\cdot)$ can be defined as

$$
\beta \odot \mathrm{m}(A) \triangleq \mathrm{m}^{\beta}(A)= \begin{cases}\beta \mathrm{m}(A), & \text { for } A \in 2^{\Theta} \\ 1-\beta, & \text { for } A=\Omega\end{cases}
$$

where, $\Omega$ is the power set of $\Theta$, i.e. $\Omega=2^{\Theta}$.

Remark 3 In this new discounting operation, $\mathrm{m}^{\beta}(\Omega)$ is not interpreted as the mass committed to the conflicting information (as $\mathrm{m}^{\alpha}(\Theta)$ in reliability discounting), nor as the mass committed to some unknown elements (as $\mathrm{m}(\emptyset)$ in Smets' open-word assumption (Smets 1990)), but only the the indecisiveness among the subset of $2^{\Theta}$ that needs to be jointly assigned with other new importance discounted sources of evidence. Further, the indecisiveness introduced in this paper is different from the non-specificity also discussed in DST literature (Beynon 2005b). It is clear that indecisiveness is defined with respect to different focal elements $A \subseteq \Theta$ (or $A \in \Omega=2^{\Theta}$ ), while non-specificity is defined with respect to different hypotheses $\theta \in \Theta$.

It is worth noting that, after the importance discounting operation, the original BBA is extended with new belief assignment $\mathrm{m}^{\beta}(\Omega)$. To facilitate later discussion, we define this kind of extended BBA as follows.

Definition 4 (importance basic belief assignment) An importance basic belief assignment (IBBA, for short) in DST framework is a function $\mathrm{m}^{\beta}(\cdot): 2^{\Theta} \cup\{\Omega\} \triangleq$ $2^{\Theta^{+}} \rightarrow[0,1]$, satisfying

$$
\left\{\begin{array}{l}
\mathrm{m}^{\beta}(\emptyset)=0 \\
\sum_{A \in 2^{\Theta}} \mathrm{m}^{\beta}(A)=\sum_{A \in 2^{\Theta}} \mathrm{m}^{\beta}(A)+\mathrm{m}^{\beta}(\Omega)=1 .
\end{array}\right.
$$


An IBBA $\mathrm{m}^{\beta}(A)$ represents the importance discounted degree of belief assigned to a proposition $A$ and measures how strongly the proposition is supported by evidence in the sense of the relative importance. The belief assigned to $\Omega$, or $\mathrm{m}^{\beta}(\Omega)$, is referred to as the degree that is not assigned to any subset of $2^{\Theta}$ and needs to be jointly assigned with other new IBBAs.

\subsection{Extended Dempster's rule of combination}

After all the sources of evidence are discounted according to the importance discounting operation defined in the above subsection, a combination rule is needed to integrate all the discounted sources to get the fusion result. As new belief assignment $\mathrm{m}^{\beta}(\Omega)$ is introduced in IBBA, so the classical Dempster's rule of combination needs to be extended to combine different IBBAs.

Definition 5 (extended Dempster's rule of combination) With two pieces of evidence with importance factors $\beta_{1}$ and $\beta_{2}$ represented by two IBBAs $\mathrm{m}_{1}^{\beta_{1}}(\cdot)$ and $\mathrm{m}_{2}^{\beta_{2}}(\cdot)$ defined on $2^{\Theta^{+}}$, the classical Dempster's rule of combination can be extended as

$$
\mathrm{m}_{1}^{\beta_{1}} \oplus \mathrm{m}_{2}^{\beta_{2}}(A) \triangleq \mathrm{m}_{12}^{\mathrm{ED}}(A)= \begin{cases}0, & \text { for } A=\emptyset \\ \frac{\sum_{B, C \in 2^{\Theta}}{ }_{; B \cap C=A} \mathrm{~m}_{1}^{\beta_{1}}(B) \mathrm{m}_{2}^{\beta_{2}}(C)}{1-\sum_{B, C \in \Theta^{\Theta}} \sum_{B \cap C=\emptyset} \mathrm{m}_{1}^{\beta_{1}}(B) \mathrm{m}_{2}^{\beta_{2}}(C)}, & \text { for } A \in 2^{\Theta^{+}} \text {and } A \neq \emptyset .\end{cases}
$$

Similar with the Dempster's rule of combination, the proposed extended Dempster's rule of combination is also based on the orthogonal sum operation $\oplus$, so the extended Dempster's rule of combination is also both commutative and associative. As a result, for multiple sources of evidence, the combination process can proceed in a recursive way to avoid high computation burden.

Lemma 1 The combination result with the extended Dempster's rule of combination is an IBBA. In other words, for the combination result, $m^{E D}(\emptyset)=0$ and $\sum_{A \in 2^{\Theta}{ }^{+}} m^{E D}(A)=$ 1.

Proof See Appendix A.

Since we usually need to work with normal BBA for decision-making support, after combining all the importance discounted IBBAs using the extended Dempster's rule of combination, the fusion result $\mathrm{m}^{\mathrm{ED}}(\cdot)$ will be normalized by redistributing the mass of belief committed to the set $\Omega$ to the other focal elements proportionally to their masses as follows

$$
\mathrm{m}^{\mathrm{norm}}(A)=\frac{\mathrm{m}^{\mathrm{ED}}(A)}{1-\mathrm{m}^{\mathrm{ED}}(\Omega)}, \forall A \in 2^{\Theta} .
$$




\section{Analysis of reliability and importance}

In the previous section, the importance of a source of evidence is defined differently from the reliability and then a method is proposed to combine sources of evidence with importance. However, the necessity of distinguishing reliability and importance in the evidence combination process is still unclear. In this section, three evidence combination axioms are proposed and explored to uncover the essential differences between reliability and importance and thus lay theoretical foundation for the proposed method.

Inclined to developing theoretically sound methods for dealing with MADM problems under uncertainty, Yang and Xu (2002) proposed four self-evident rules, with which a rational attribute aggregation process needs to satisfy. Inspired by these ideas, here we refine and extend these rules within the framework of DST and propose three evidence combination axioms as follows $\left(\operatorname{Suppose~}_{i}(\cdot)(i=1, \cdots, L)\right.$ are $L$ basic sources of evidence's BBAs on the same frame of discernment $\Theta$ and $\mathrm{m}(\cdot)$ is the fusion result).

Axiom 1 (independence) If no evidence assigns mass to one non-global ignorance set or its supersets, then the combination result will not assign mass to the same set either. Mathematically, $\forall S \in 2^{\Theta} \backslash \Theta$, if $\forall S^{+} \supseteq S, m_{i}\left(S^{+}\right)=0$ for all $i=1, \cdots, L$, then $m(S)=0$.

Axiom 2 (consensus) If all evidence assigns full mass to one non-global ignorance set, then the combination result will assign full mass to the same set too. Mathematically, $\forall S \in 2^{\Theta} \backslash \Theta$, if $m_{i}(S)=1$ for all $i=1, \cdots, L$, then $m(S)=1$.

Axiom 3 (completeness) If all evidence assigns full mass to the subsets of one nonglobal ignorance set, the combination result will also assign full mass to the subsets of the same set. Mathematically, $\forall S \in 2^{\Theta} \backslash \Theta$, if $\sum_{S^{-} \subseteq S} m_{i}\left(S^{-}\right)=1$ for all $i=1, \cdots, L$, then $\sum_{S^{-} \subseteq S} m\left(S^{-}\right)=1$.

Remark 4 In the three axioms defined above, the global ignorance set $\Omega$ is not taken into consideration mainly because as a special set it may take other explanation (e.g. conflicting information in reliability discounting) besides global ignorance. So, the explanation ambiguity of set $\Omega$ will destroy the rationality of the three axioms. Besides, the three axioms are extended from Yang's self-evident rules (Yang and $\mathrm{Xu} 2002$ ), in which they also didn't take into account the global ignorance set in the attribute aggregation process.

In order to explain the three evidence combination axioms in a more comprehensible way, a simple MADM example is given below.

Example 1 (car performance evaluation). As in Fig.1, we consider the problem of evaluating the performance of three types of car (decision alternatives, DAs), namely, $A, B$, and $C$. In the DST terminology used above, $\{A, B, C\}$ is the frame of discernment $\Theta$. The overall performance of each type of car is evaluated based on three major attributes: quality of engine, operation, and comfortableness. Now, the three axioms can be explained from the aspect of MADM as follows.

- Suppose no attribute assigns belief to car A as the best car, then the attribute aggregation result will not assign any belief to car A. (independence) 


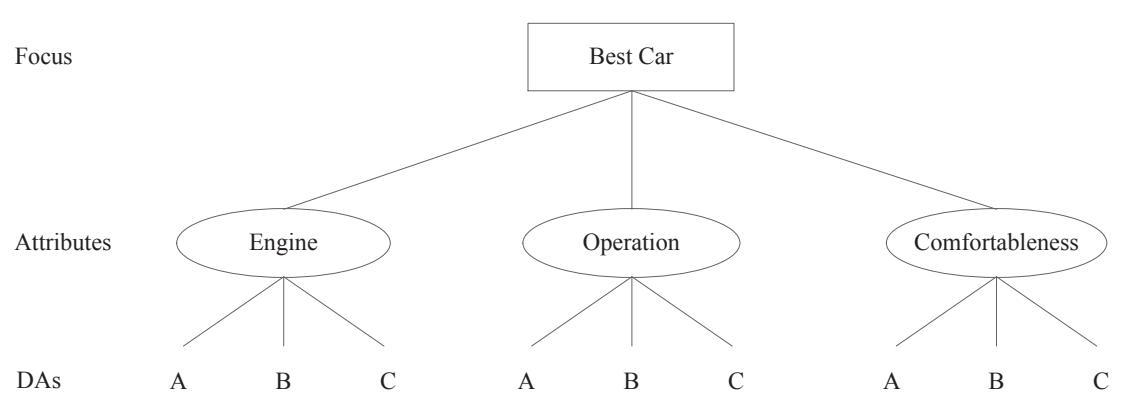

Fig. 1 A car performance evaluation example.

- Suppose all attributes assign full belief to car A as the best car, then the attribute aggregation result will also assign full belief to car A. (consensus)

- Suppose all attributes assign full belief to car B or C as the best car, then the attribute aggregation result will also assign full belief to car B or C. (completeness)

The above axioms characterize three commonsense properties of the evidence combination process. While, if the reliability of sources of evidence is considered, some of them may not be satisfied as shown in the following theorem.

Theorem 1 The reliability discounting and combination method, displayed as Eqs.(3)(4), satisfies the independence axiom, but fails to satisfy the consensus and completeness axioms.

\section{Proof See Appendix B.}

This theorem is straightforward if one has a deep understanding of the reliability. Now, we consider the consensus axiom in Example 1. Suppose all attributes assign full belief to car A as the best car, considering the decision maker is unfamiliar with the engine, wrong assessment may be provided (maybe car B has the best engine performance in reality), and so the attribute aggregation result should not assign full belief to car A as the best car.

Then, with different concept from reliability, it's interesting to know whether the importance discounting and combination method proposed in Sect. 3 satisfies the three axioms. The following theorem presents the conclusion.

Theorem 2 The proposed importance discounting and combination method, displayed as Eqs.(5),(7)-(8), satisfies the independence, consensus and completeness axioms.

Proof See Appendix C.

As illustrated in the above theorem, distinct from the reliability, the consensus and completeness axioms are still preserved if the importance is considered in the evidence combination process. Here, we also take the consensus axiom for example to explain it in a reasonable way. Suppose all attributes assign full belief to car A as the best car, as the importance is a subjective property of a source of evidence and it does no matter with the source's capability, so the importance discounting only assign weights to each attributes rather than change their assessments, and 
thus it's intuitively reasonable that the attribute aggregation result will also assign full belief to car A.

In short, due to the fact that reliability and importance are distinct notions, they should be managed in different ways and consequently the two discounting and combination methods differ in properties. Furthermore, considering that reliability and importance may coexist in some cases, a general scheme for combination of sources of evidence will be developed in next section.

\section{A general scheme for combination of sources of evidence}

In this section, we would like to establish a general scheme for combination of sources of evidence. Now, we examine the possibility to take into account both the reliability factor $\alpha$ and the importance factor $\beta$ of a given source of evidence characterized by its BBA $\mathrm{m}(\cdot)$. Apparently, it's a natural way to discount the original BBA $\mathrm{m}(\cdot)$ by $\alpha$ with reliability discounting operation and then discount the result $\mathrm{m}^{\alpha}(\cdot)$ by $\beta$ with importance discounting operation. This kind of realization is given as follows.

Definition 6 (reliability-importance discounting operation) We define the reliabilityimportance discounting operation of a source of evidence having the reliability factor $\alpha \in[0,1]$, the importance factor $\beta \in[0,1]$ and the associated BBA $\mathrm{m}(\cdot)$ in the frame of discernment $\Theta$ by

$$
\beta \odot(\alpha \otimes \mathrm{m}(A)) \triangleq \mathrm{m}^{\alpha, \beta}(A)= \begin{cases}\alpha \beta \mathrm{m}(A), & \text { for } A \in 2^{\Theta}, A \neq \Theta \\ \alpha \beta \mathrm{m}(\Theta)+(1-\alpha) \beta, & \text { for } A=\Theta \\ 1-\beta, & \text { for } A=\Omega .\end{cases}
$$

where, $\Omega$ has the same meaning as in Definition 3 .

As the result of reliability-importance discounting operation is an IBBA, so the proposed extended Dempster's rule of combination in Sect. 3 can be used to combine multiple reliability-importance discounted IBBAs.

This kind of reliability-importance discounting and combination method provides a general scheme for combination of sources of evidence to take into account both the reliability and importance. It can be seen that, when $\beta=1$ (the source has full importance), the reliability-importance discounting and combination method simplifies to the reliability discounting and combination method presented in Sect. 2 ; when $\alpha=1$ (the source has full reliability), the importance discounting and combination method proposed in Sect. 3 is obtained; and when both $\alpha=1$ and $\beta=1$ (the source has both full importance and reliability), the original BBA $\mathrm{m}(\cdot)$ keeps unchanged and the classical Dempster's rule of combination is used to integrate the sources of evidence.

Remark 5 Because the reliability is considered in the evidence combination process, it's easy to know that the proposed general scheme also only satisfies the independence axiom, and it doesn't satisfy the consensus and completeness axioms except that all the sources of evidence take full reliability $\left(\alpha_{i}=1, i=1, \cdots, L\right)$, in which case the general scheme reduces to the importance discounting and combination method. 


\section{Case study}

\subsection{Problem presentation}

Here we conduct a comprehensive study of the car performance evaluation problem described in Example 1. For this MADM problem, as described in Sect. 3, the relative importance of different attributes plays significant role in the process of decision making. Besides, the knowledge level of decision maker (DM) may vary in the three corresponding fields (i.e. Engine, Operation, and Comfortableness), and so the reliability of different knowledge matrices provided by the DM should also be considered in the decision process.

The DS/AHP framework (Beynon et al 2000; Beynon 2002) is used here to conduct the analysis of the above MADM problem. Compared with standard analytic hierarchy process (AHP) method, the inclusion of DST allows for uncertainty in the judgements. More details about this framework can be found in (Beynon et al 2000). Within the DS/AHP framework, it is assumed that with respect to each attribute the "uncertain" knowledge matrices for decision alternatives (DAs) are given by the DM based on his/her knowledge as Table 1.

Table 1 Knowledge matrices for different attributes

\begin{tabular}{lllll}
\hline Engine & $A$ & $B$ & $C$ & $\Theta$ \\
\hline$A$ & 1 & $0^{a}$ & 0 & 3 \\
$B$ & 0 & 1 & 0 & $1 / 2$ \\
$C$ & 0 & 0 & 1 & 1 \\
$\Theta$ & $1 / 3$ & 2 & 1 & 1 \\
\hline \hline Operation & $A$ & $B$ & $C$ & $\Theta$ \\
\hline$A$ & 1 & 0 & 0 & 2 \\
$B$ & 0 & 1 & 0 & 5 \\
$C$ & 0 & 0 & 1 & 1 \\
$\Theta$ & $1 / 2$ & $1 / 5$ & 1 & $\Theta$ \\
\hline \hline Comfortableness & $A$ & $B$ & $C$ & $1 / 2$ \\
\hline$A$ & 1 & 0 & 0 & 1 \\
$B$ & 0 & 1 & 0 & 2 \\
$C$ & 0 & 0 & 1 & 1 \\
\hline
\end{tabular}

a The zero's appearing in the knowledge matrix indicate there is no necessity to compare different individual DAs (e.g., A to B) directly; this assertion can be made indirectly through knowledge of the favorability of $\mathrm{A}$ to $\Theta$ and $\mathrm{B}$ to $\Theta$ relatively.

6.2 Derivation of BBAs, reliability and importance factors

The derivation of BBAs is the basis for evidence combination in DST. Here, the method in (Beynon et al 2000) is used to construct the BBAs from the above 
knowledge matrices. The normalized principal eigenvectors of the knowledge matrices displayed in Table 1 are derived as the corresponding BBAs for the three attributes engine, operation and comfortableness as follows.

$$
\begin{array}{lllll}
\mathrm{m}_{1}(\cdot): & \mathrm{m}_{1}(A)=0.4814, & \mathrm{~m}_{1}(B)=0.0802, & \mathrm{~m}_{1}(C)=0.1605, & \mathrm{~m}_{1}(\Theta)=0.2779 \\
\mathrm{~m}_{2}(\cdot): & \mathrm{m}_{2}(A)=0.2055, & \mathrm{~m}_{2}(B)=0.5138, & \mathrm{~m}_{2}(C)=0.1028, & \mathrm{~m}_{2}(\Theta)=0.1780 \\
\mathrm{~m}_{3}(\cdot): & \mathrm{m}_{3}(A)=0.0956, & \mathrm{~m}_{3}(B)=0.1911, & \mathrm{~m}_{3}(C)=0.3823, & \mathrm{~m}_{3}(\Theta)=0.3310
\end{array}
$$

The reliability factors will be estimated if the DM's knowledge level in the three corresponding fields is known. For example, if it is known prior that the DM is familiar with engine, expert in operation but has a low sensitivity for the comfortableness of a car, then the reliability factors for the above three BBAs can be empirically set as $\alpha_{1}=0.9, \alpha_{2}=1.0$ and $\alpha_{3}=0.5$, respectively.

As for the importance factors, the AHP method mentioned in Sect. 3 is utilized here. Suppose the DM believes that comfortableness is two times more important than the quality of engine, three times more important than the quality of operation and the quality of engine is two times more important than the quality of

\begin{tabular}{|c|c|c|c|}
\hline Engine & 1 & 2 & $1 / 2$ \\
\hline Operation & $1 / 2$ & 1 & $1 / 3$ \\
\hline Comfortableness & 2 & 3 & 1 \\
\hline
\end{tabular}
operation, then the preference matrix among the three attributes is displayed as

Engine Operation Comfortableness

Its normalized principal eigenvector $w=[0.30,0.16,0.54]$ can be used as the corresponding importance factors.

\subsection{Numerical results}

In this numerical study, to make a comparison, the reliability discounting and combination method (RDC, only consider the reliability), the importance discounting and combination method (IDC, only consider the importance), and the reliability-importance discounting and combination method (RIDC, consider both the reliability and importance) are tested to integrate the BBAs with differen$\mathrm{t}$ reliability and importance factors derived in the previous subsection. For each method, the BBAs for the three attributes are firstly discounted and then combined to get the combination result. The final decision is then can be made based on the combination result according to some decision criteria.

Table 2 Combination results with different methods(RDC, IDC, and RIDC)

\begin{tabular}{lllllll}
\hline & $\mathrm{m}_{1}$ & $\mathrm{~m}_{2}$ & $\mathrm{~m}_{3}$ & RDC & IDC & RIDC \\
\hline$\{A\}$ & 0.4814 & 0.2055 & 0.0956 & 0.3354 & 0.2260 & 0.1905 \\
$\{B\}$ & 0.0802 & 0.5138 & 0.1911 & 0.4046 & 0.2161 & 0.1674 \\
$\{C\}$ & 0.1605 & 0.1028 & 0.3823 & 0.1618 & 0.2974 & 0.1740 \\
$\Theta$ & 0.2779 & 0.1780 & 0.3310 & 0.0982 & 0.2605 & 0.4681 \\
\hline
\end{tabular}

Table 2 shows the combination results of the three pieces of evidence using different methods (Eqs.(3)-(4) for RDC, Eqs.(5),(7)-(8) for IDC, Eqs.(9),(7)-(8) for 
RIDC, respectively). Usually, the DM is concerned with a single choice among the three cars. The decision can be made using the maximum of pignistic probability (BetP) criteria defined as Eq.(2) over the raw combination results obtained above.

Table 3 BetP criteria and decision results based on BetP

\begin{tabular}{lllllll}
\hline Cars & Engine & Operation & Comfortableness & RDC & IDC & RIDC \\
\hline$A$ & 0.5740 & 0.2648 & 0.2059 & 0.3681 & 0.3128 & 0.3465 \\
$B$ & 0.1729 & 0.5731 & 0.3015 & 0.4374 & 0.3030 & 0.3234 \\
$C$ & 0.2531 & 0.1621 & 0.4926 & 0.1945 & 0.3842 & 0.3301 \\
\hline \multirow{2}{*}{ Decision } & $A \succ C \succ B$ & $B \succ A \succ C$ & $C \succ B \succ A$ & $B \succ A \succ C$ & $C \succ A \succ B$ & $A \succ C \succ B$ \\
\hline
\end{tabular}

Table 3 shows the BetP criteria of the DAs (Rows 2-4) as well as the decision results based on these criteria (Row 5). For comparison, apart from the three methods (RDC, IDC, and RIDC), the decision results based on each individual attribute (Engine, Operation, and Comfortableness) are also given. It can be seen that the decision results are different for the three methods mainly because different kinds of additional information are considered in the decision process. For RDC method, the decision result is the same with that based on the Operation attribute, since its corresponding knowledge matrix has the largest reliability. For IDC method, the decision result about the best car is the same with that based on the Comfortableness attribute, since the DM assigns the greatest importance to this attribute. While, for RIDC method, the decision result is the same with that based on the Engine attribute which behaves not bad both in reliability and importance. It's believed that the RIDC method gives the most reasonable decision result as both the reliability of the knowledge matrices and the importance of attributes are considered.

\section{Conclusions}

Real-world fusion problems are complex in that they often involve multiple sources of evidence with uncertainty. Further, different sources of evidence to be combined may not have the same reliability or importance, while the two properties make no difference in some previous works. In this paper, the concepts of reliability and importance are clarified and the importance discounting and combination method composed of an importance discounting operation and an extended Dempster's rule of combination is proposed within the framework of DST to integrate sources of evidence with importance. We show some interesting axioms satisfied by the new method, which further reveal the essential differences between reliability and importance. A general scheme is developed to combine sources of evidence to take into account both the importance and reliability. An example of car performance evaluation is studied and the numerical results show the efficiency of the new general scheme considering both the reliability of the knowledge matrices and the importance of different attributes. 
Noting that the importance of a source of evidence is still a new concept in DST framework, the importance discounting and combination method proposed in this paper is just one kind of realization. That is, other importance discounting and combination methods in future works may also be reasonable if some axioms (such as independence, consensus and completeness) reflecting the nature of importance are satisfied.

In addition, this paper mainly focuses on the combination of sources of evidence with reliability and importance encountered in general MADM problems. While, to the more complicated group multi-attribute decision making (GMADM) problems (Beynon 2005a), besides the attributes' reliability and importance considered in general MADM, a rational evidence aggregation process also needs to take into account the importance of individuals in the group. In future work, we will extend the reliability-importance discounting and combination method developed in this paper to address the evidence aggregation problem in GMADM.

\section{A Proof of Lemma 1}

According to Definition $5, \mathrm{~m}_{12}^{\mathrm{ED}}(\emptyset)=0$ is satisfied directly. Besides,

$$
\begin{aligned}
\sum_{A \in 2^{\Theta}} \mathrm{m}_{12}^{\mathrm{ED}}(A) & =\sum_{A \in 2^{\Theta^{+}}, A \neq \emptyset} \mathrm{m}_{12}^{\mathrm{ED}}(A)+\mathrm{m}_{12}^{\mathrm{ED}}(\emptyset)=\sum_{A \in 2^{\Theta^{+}}, A \neq \emptyset} \mathrm{m}_{12}^{\mathrm{ED}}(A) \\
= & \frac{\sum_{A \in 2^{\Theta^{+}}, A \neq \emptyset}\left(\sum_{B, C \in 2^{\Theta^{+}} ; B \cap C=A} \mathrm{~m}_{1}^{\beta_{1}}(B) \mathrm{m}_{2}^{\beta_{2}}(C)\right)}{1-\sum_{B, C \in 2^{\Theta^{+}} ; B \cap C=\emptyset} \mathrm{m}_{1}^{\beta_{1}}(B) \mathrm{m}_{2}^{\beta_{2}}(C)} .
\end{aligned}
$$

Because,

$$
\begin{aligned}
& \sum_{A \in 2^{\Theta^{+}}, A \neq \emptyset}\left(\sum_{B, C \in 2^{\Theta^{+}} ; B \cap C=A} \mathrm{~m}_{1}^{\beta_{1}}(B) \mathrm{m}_{2}^{\beta_{2}}(C)\right)+\sum_{B, C \in 2^{\Theta^{+}} ; B \cap C=\emptyset} \mathrm{m}_{1}^{\beta_{1}}(B) \mathrm{m}_{2}^{\beta_{2}}(C) \\
= & \sum_{A \in 2^{\Theta}}\left(\sum_{B, C \in 2^{\Theta^{+}} ; B \cap C=A} \mathrm{~m}_{1}^{\beta_{1}}(B) \mathrm{m}_{2}^{\beta_{2}}(C)\right)=\sum_{B \in 2^{\Theta^{+}}}\left(\mathrm{m}_{1}^{\beta_{1}}(B) \sum_{C \in 2^{\Theta}} \mathrm{m}_{2}^{\beta_{2}}(C)\right) \\
= & \sum_{B \in 2^{\Theta}} \mathrm{m}_{1}^{\beta_{1}}(B)=1,
\end{aligned}
$$

so, Eq.(10) equals 1. Therefore, the combination result $\mathrm{m}_{12}^{\mathrm{ED}}(\cdot)$ with the extended Dempster's rule of combination is an IBBA.

\section{B Proof of Theorem 1}

Suppose $\mathrm{m}_{i}(\cdot)(i=1, \cdots, L)$ are $L$ basic sources of evidence's BBAs on the same frame of discernment $\Theta$ with reliability factors $\alpha_{i} \in[0,1](i=1, \cdots, L)$. Denote $\mathrm{m}(\cdot)$ the integrated BBA with the reliability discounting and combination method.

As for the independence axiom, $\forall S \in 2^{\Theta} \backslash \Theta$, suppose $\forall S^{+} \supset S, \mathrm{~m}_{i}\left(S^{+}\right)=0$ for all $i=1, \cdots, L$. Discount all the $L$ BBAs with their corresponding reliability factors $\alpha_{i}$ using Shafer's discounting operation displayed as Eq.(3), we can get the reliability discounted BBAs assigned to $S^{+}$

$$
\mathrm{m}_{i}{ }^{\alpha_{i}}(A)= \begin{cases}0, & \text { for } A \in 2^{\Theta}, A \supseteq S, A \neq \Theta \quad \text { for } \quad i=1, \cdots, L . \\ 1-\alpha_{i}, & \text { for } A=\Theta\end{cases}
$$


Then, the Dempster's rule of combination displayed as Eq.(4) will be used to get the integrated BBAs assigned to $S$

$$
\mathrm{m}(S)=\sum_{X_{i} \in 2^{\Theta} ; \bigcap_{i=1}^{L} X_{i}=S} \prod_{i=1}^{L} \mathrm{~m}_{i}^{\alpha_{i}}\left(X_{i}\right) /\left(1-\sum_{X_{i} \in 2^{\Theta} ; \bigcap_{i=1}^{L} X_{i}=\emptyset} \prod_{i=1}^{L} \mathrm{~m}_{i}^{\alpha_{i}}\left(X_{i}\right)\right) .
$$

Since $\bigcap_{i=1}^{L} X_{i}=S$, so $X_{i} \supseteq S$. According to Eq.(11), if $X_{i} \neq \Theta, \mathrm{m}_{i}^{\alpha_{i}}\left(X_{i}\right)=0$ for all $i=1, \cdots, L$. Because it's impossible for all $X_{i}$ to take $\Theta$ satisfying $\bigcap_{i=1}^{L} X_{i}=S$, so, $\prod_{i=1}^{L} \mathrm{~m}_{i}^{\alpha_{i}}\left(X_{i}\right)$ will equal 0 in any cases. Hence, $\mathrm{m}(S)$ in Eq.(12) equals 0. That is, the reliability discounting and combination method satisfies the independence axiom.

As for the consensus axiom, $\forall S \in 2^{\Theta} \backslash \Theta$, suppose $\mathrm{m}_{i}(S)=1$ for all $i=1, \cdots, L$. Discount all the $L$ BBAs with their corresponding reliability factors $\alpha_{i}$ using Shafer's discounting operation displayed as Eq.(3), we can get the reliability discounted BBAs

$$
\mathrm{m}_{i}{ }^{\alpha_{i}}(A)= \begin{cases}\alpha_{i}, & \text { for } A=S \\ 0, & \text { for } A \in 2^{\Theta}, A \neq S, A \neq \Theta \quad \text { for } i=1, \cdots, L \\ 1-\alpha_{i}, & \text { for } A=\Theta\end{cases}
$$

Then, the Dempster's rule of combination displayed as Eq.(4) will be used to integrate the reliability discounted BBAs

$$
\mathrm{m}(A)= \begin{cases}0, & \text { for } A \in 2^{\Theta}, A \neq S, A \neq \Theta \\ \prod_{i=1}^{L}\left(1-\alpha_{i}\right), & \text { for } A=\Theta\end{cases}
$$

So,

$$
\mathrm{m}(S)=1-\sum_{A \in 2^{\Theta}, A \neq S, A \neq \Theta} \mathrm{m}(A)-\mathrm{m}(\Theta)=1-\prod_{i=1}^{L}\left(1-\alpha_{i}\right)
$$

Thus, the reliability discounting and combination method only satisfies the consensus axiom when at least one source of evidence takes full reliability $\left(\exists k \in\{1, \cdots, L\}, \alpha_{k}=1\right)$.

As for the completeness axiom, $\forall S \in 2^{\Theta} \backslash \Theta$, suppose $\sum_{S-\subseteq S} \mathrm{~m}_{i}\left(S^{-}\right)=1$ for all $i=$ $1, \cdots, L$. We can know that $\forall S^{++} \supset S, \mathrm{~m}_{i}\left(S^{++}\right)=0$. Discount all the $L$ BBAs with their corresponding reliability factors $\alpha_{i}$ using Shafer's discounting operation displayed as Eq.(3), we can get the reliability discounted BBAs assigned to $S^{++}$

$$
\mathrm{m}_{i}{ }^{\alpha_{i}}(A)=\left\{\begin{array}{ll}
0, & \text { for } A \in 2^{\Theta}, A \supset S, A \neq \Theta \\
1-\alpha_{i}, & \text { for } A=\Theta
\end{array} \text { for } i=1, \cdots, L .\right.
$$

Then, the Dempster's rule of combination displayed as Eq.(4) will be used to get the integrated BBAs assigned to $S^{++}$

$$
\mathrm{m}\left(S^{++}\right)=\sum_{X_{i} \in 2^{\Theta} ; \bigcap_{i=1}^{L} X_{i}=S^{++}} \prod_{i=1}^{L} \mathrm{~m}_{i}^{\alpha_{i}}\left(X_{i}\right) /\left(1-\sum_{X_{i} \in 2^{\Theta} ; \prod_{i=1}^{L} X_{i}=\emptyset} \prod_{i=1}^{L} \mathrm{~m}_{i}^{\alpha_{i}}\left(X_{i}\right)\right)
$$

Since $\bigcap_{i=1}^{L} X_{i}=S^{++}$, so $X_{i} \supseteq S^{++} \supset S$. Now, we consider it for two cases. If $S^{++} \neq \Theta$, according to Eq.(13), $\mathrm{m}_{i}^{\alpha_{i}}\left(X_{i}\right)=0$ for all $i=1, \cdots, L$. So, $\mathrm{m}\left(S^{++}\right)$in Eq.(14) equals 0. If $S^{++}=\Theta$, it's easy to get $\mathrm{m}\left(S^{++}\right)=\mathrm{m}(\Theta)=\prod_{i=1}^{L}\left(1-\alpha_{i}\right) /(1-k)$. 
So,

$\sum_{S^{-} \subseteq S} \mathrm{~m}\left(S^{-}\right)=1-\sum_{S^{++} \supset S} \mathrm{~m}\left(S^{++}\right)=1-\sum_{S^{++} \supset S, S^{++} \neq \Theta} \mathrm{m}\left(S^{++}\right)-\mathrm{m}(\Theta)$

$=1-\mathrm{m}(\Theta)=1-\prod_{i=1}^{L}\left(1-\alpha_{i}\right) /(1-k)$.

Therefore, the reliability discounting and combination method only satisfies the completeness axiom when at least one source of evidence takes full reliability $\left(\exists k \in\{1, \cdots, L\}, \alpha_{k}=1\right)$.

\section{Proof of Theorem 2}

Suppose $\mathrm{m}_{i}(\cdot)(i=1, \cdots, L)$ are $L$ basic sources of evidence's BBAs on the same frame of discernment $\Theta$ with importance factors $\beta_{i} \in[0,1](i=1, \cdots, L)$. Denote $\mathrm{m}(\cdot)$ the integrated BBA with the proposed importance discounting and combination method.

As for the independence axiom, $\forall S \in 2^{\Theta} \backslash \Theta$, suppose $\forall S^{+} \supseteq S, \mathrm{~m}_{i}\left(S^{+}\right)=0$ for all $i=1, \cdots, L$. Discount all the $L$ BBAs with their corresponding importance factors $\beta_{i}$ using the importance discounting operation displayed as Eq.(5), we can get the importance discounted IBBAs assigned to $S^{+}$

$$
\mathrm{m}_{i}{ }^{\beta_{i}}(A)=0, \forall A \supseteq S, \text { for } i=1, \cdots, L .
$$

Then, the extended Dempster's rule of combination displayed as Eq.(7) will be used to get the integrated IBBAs assigned to $S$

$$
\mathrm{m}^{\mathrm{ED}}(S)=\sum_{X_{i} \in 2^{\Theta+} ; \bigcap_{i=1}^{L} X_{i}=S} \prod_{i=1}^{L} \mathrm{~m}_{i}^{\beta_{i}}\left(X_{i}\right) /\left(1-\sum_{X_{i} \in 2^{\Theta+} ; \bigcap_{i=1}^{L} X_{i}=\emptyset} \prod_{i=1}^{L} \mathrm{~m}_{i}^{\beta_{i}}\left(X_{i}\right)\right) .
$$

As $\bigcap_{i=1}^{L} X_{i}=S$, so $X_{i} \supseteq S$. According to Eq.(15), $\mathrm{m}_{i}^{\beta_{i}}\left(X_{i}\right)=0$ for all $i=1, \cdots, L$. Hence, $\mathrm{m}^{\mathrm{ED}}(S)$ in Eq.(16) equals 0. It's straightforward that $\mathrm{m}(S)=\mathrm{m}^{\mathrm{ED}}(S) /\left(1-\mathrm{m}^{\mathrm{ED}}(\Omega)\right)=0$. That is, the importance discounting and combination method satisfies the independence axiom.

As for the consensus axiom, $\forall S \in 2^{\Theta} \backslash \Theta$, suppose $\mathrm{m}_{i}(S)=1$ for all $i=1, \cdots, L$. Discount all the $L$ BBAs with their corresponding importance factors $\beta_{i}$ using the importance discounting operation displayed as Eq.(5), we can get the importance discounted IBBAs

$$
\mathrm{m}_{i}{ }^{\beta_{i}}(A)= \begin{cases}\beta_{i}, & \text { for } A=S \\ 0, & \text { for } A \in 2^{\Theta}, A \neq S \quad \text { for } i=1, \cdots, L . \\ 1-\beta_{i}, & \text { for } A=\Omega\end{cases}
$$

Then, the extended Dempster's rule of combination displayed as Eq.(7) will be used to integrate the importance discounted IBBAs

$$
\mathrm{m}^{\mathrm{ED}}(A)=0, \quad \forall A \in 2^{\Theta}, A \neq S .
$$

According to Lemma 1, it holds that

$$
\sum_{A \in 2^{\Theta^{+}}} \mathrm{m}^{\mathrm{ED}}(A)=\sum_{A \in 2^{\Theta}, A \neq S} \mathrm{~m}^{\mathrm{ED}}(A)+\mathrm{m}^{\mathrm{ED}}(S)+\mathrm{m}^{\mathrm{ED}}(\Omega)=\mathrm{m}^{\mathrm{ED}}(S)+\mathrm{m}^{\mathrm{ED}}(\Omega)=1 .
$$

Furthermore, via the normalization in Eq.(8), we obtain

$$
\mathrm{m}(S)=\frac{\mathrm{m}^{\mathrm{ED}}(S)}{1-\mathrm{m}^{\mathrm{ED}}(\Omega)}=\frac{\mathrm{m}^{\mathrm{ED}}(S)}{\mathrm{m}^{\mathrm{ED}}(S)}=1 .
$$

So, the importance discounting and combination method satisfies the consensus axiom. 
As for the completeness axiom, $\forall S \in 2^{\Theta} \backslash \Theta$, suppose $\sum_{S^{-} \subseteq S} \mathrm{~m}_{i}\left(S^{-}\right)=1$ for all $i=$ $1, \cdots, L$. We can know that $\forall S^{++} \supset S, \mathrm{~m}_{i}\left(S^{++}\right)=0$. Discount all the $L$ BBAs with their corresponding importance factors $\beta_{i}$ using the importance discounting operation displayed as Eq.(5), we can get the importance discounted IBBAs assigned to $S^{++}$

$$
\mathrm{m}_{i}{ }^{\beta_{i}}(A)=0, \forall A \supset S, \text { for } i=1, \cdots, L .
$$

Then, the extended Dempster's rule of combination displayed as Eq.(7) will be used to get the integrated IBBAs assigned to $S^{++}$

$$
\mathrm{m}^{\mathrm{ED}}\left(S^{++}\right)=\sum_{X_{i} \in 2^{\Theta+}} \sum_{i=1}^{L} \prod_{i=1}^{L} \mathrm{~m}_{i}^{\beta_{i}}\left(X_{i}\right) /\left(1-\sum_{X_{i} \in 2^{\Theta+} ; \bigcap_{i=1}^{L} X_{i}=\emptyset} \prod_{i=1}^{L} \mathrm{~m}_{i}^{\beta_{i}}\left(X_{i}\right)\right) .
$$

As $\bigcap_{i=1}^{L} X_{i}=S^{++}$, so $X_{i} \supseteq S^{++} \supset S$. According to Eq.(17), $\mathrm{m}_{i}^{\beta_{i}}\left(X_{i}\right)=0$ for all $i=1, \cdots, L$. Hence, $\mathrm{m}^{\mathrm{ED}}\left(S^{++}\right)$in Eq.(18) equals 0. It's straightforward that $\mathrm{m}\left(S^{++}\right)=$ $\mathrm{m}^{\mathrm{ED}}\left(S^{++}\right) /\left(1-\mathrm{m}^{\mathrm{ED}}(\Omega)\right)=0$. So, $\sum_{S^{-} \subseteq S} \mathrm{~m}\left(S^{-}\right)=1-\sum_{S^{++} \supset S} \mathrm{~m}\left(S^{++}\right)=1$. That is, the importance discounting and combination method satisfies the completeness axiom.

\section{References}

Anand SS, Bell DA, et al (1996) EDM: a general framework for data mining based on evidence theory. Data Knowl Eng 18(3):189-223

Barron FH, Barrett BE (1996) Decision quality using ranked attribute weights. Manag Sci 42(11):1515-1523

Beynon M (2002) DS/AHP method: A mathematical analysis, including an understanding of uncertainty. Eur J Operat Res 140(1):148-164

Beynon M (2005a) A method of aggregation in DS/AHP for group decision-making with the non-equivalent importance of individuals in the group. Comput Operat Res 32(7):18811896

Beynon M (2005b) Understanding local ignorance and non-specificity within the DS/AHP method of multi-criteria decision making. Eur J Operat Res 163(2):403-417

Beynon M, Curry B, et al (2000) The Dempster-Shafer theory of evidence: an alternative approach to multicriteria decision modelling. OMEGA-Int J Manag Sci 28(1):37-50

Beynon M, Cosker D, et al (2001) An expert system for multi-criteria decision making using Dempster-Shafer theory. Expert Syst Appl 20(4):357-367

Davis JP, Hall JW (2003) A software-supported process for assembling evidence and handling uncertainty in decision making. Decis Support Syst 35(3):415-433

Dempster A (1967) Upper and lower probabilities induced by multivalued mapping. Ann Math Stat 38(2):325-339

Deng Y, Shi WK, et al (2004) Combining belief functions based on distance of evidence. Decis Support Syst 38(3):489-493

Denœux T (1995) A k-nearest neighbor classification rule based on Dempster-Shafer theory. IEEE T Syst Man Cy 25(5):804-813

Denœux T, Masson MH (2012) Evidential reasoning in large partially ordered sets. Ann Operat Res 195:135-161

Dijkstra TK (2013) On the extraction of weights from pairwise comparison matrices. Central Eur J Operat Res 21(1):103-123

Ghasemi J, Ghaderi R, et al (2013) A novel fuzzy Dempster-Shafer inference system for brain MRI segmentation. Inform Sci 223(5):205-220

Haenni R, Hartmann S (2006) Modeling partially reliable information sources: a general approach based on Dempster-Shafer theory. Inform Fusion 7(4):361-379

Hégarat-Mascle SL, Bloch I, et al (1998) Introduction of neighborhood information in evidence theory and application to data fusion of radar and optical images with partial cloud cover. Pattern Recogn 31(11):1811-1823 
Hwang CL, Yoon K (1981) Multiple attribute decision-making: methods and applications. Berlin, Germany: Springer

Ishizaka A, Lusti M (2006) How to derive priorities in AHP: a comparative study. Central Eur J Operat Res 14:387-400

Liu ZG, Dezert J, et al (2011) Combination of sources of evidence with different discounting factors based on a new dissimilarity measure. Decis Support Syst 52(1):133-141

Mercier D, Quost B, et al (2008) Refined modeling of sensor reliability in the belief function framework using contextual discounting. Inform Fusion 9(2):246-258

Milisavljević N, Bloch I (2003) Sensor fusion in anti-personnel mine detection using a two-level belief function model. IEEE T Syst Man Cy C 33(2):269-283

Roberts R, Goodwin P (2002) Weight approximations in multi-attribute decision models. J Multi-Crit Decis Anal 11(6):291-303

Scotney B, McClean S (2003) Database aggregation of imprecise and uncertain evidence. Inform Sci 155(3):245-263

Shafer G (1976) A Mathematical Theory of Evidence. Princeton, NJ: Princeton University Press

Shafer G (1987) Probability judgment in artificial intelligence and expert systems. Stat Sci 2(1):3-16

Smarandache F, Dezert J (2006) Proportional conflict redistribution rules for information fusion. In: Smarandache F, Dezert J (eds) Advances and Applications of DSmT for Information Fusion (Collected works), Rehoboth: American Research Press, vol 2, pp 3-68

Smarandache F, Dezert J, et al (2010) Fusion of sources of evidence with different importances and reliabilities. In: Proceedings of 13th International Conference on Informance Fusion, Edinburgh, UK

Smets P (1990) The combination of evidence in the transferable belief model. IEEE T Pattern Anal 12(2):447-458

Smets P (2005) Decision making in the TBM: the necessity of the pignistic transformation. Int J Approx Reason 38(2):133-147

Srinivasan T, Chandrasekhar A, et al (2005) Knowledge discovery in clinical databases with neural network evidence combination. In: Proceedings of ICISIP, pp 512-517

Tabassian M, Ghaderi R, et al (2012) Combining complementary information sources in the DempstercShafer framework for solving classification problems with imperfect labels. Knowl-Based Syst 27(1):92-102

Tacnet JM, Batton-Hubert M, et al (2009) Information fusion for natural hazards in mountains. In: Smarandache F, Dezert J (eds) Advances and Applications of DSmT for Information Fusion (Collected works), Rehoboth: American Research Press, vol 3, pp 365-660

Xu DL (2012) An introduction and survey of the evidential reasoning approach for multiple criteria decision analysis. Ann Operat Res 195:163-187

Xu DL, Yang JB, Wang YM (2006) The ER approach for multi-attribute decision analysis under interval uncertainties. Eur J Operat Res 174(3):1914-1943

$\mathrm{Xu} \mathrm{X}$ (2004) A note on the subjective and objective integrated approach to determine attribute weights. Eur J Operat Res 156(2):530-532

Yang JB, Sen P (1994) A general multi-level evaluation process for hybrid multiple attribute decision making with uncertainty. IEEE T Syst Man Cy 24(10):1458-1473

Yang JB, Xu DL (2002) On the evidential reasoning algorithm for multiple attribute decision analysis under uncertainty. IEEE T Syst Man Cy A 32(3):289-304

Yang JB, Xu DL (2011) Introduction to the ER rule for evidence combination. In: Tang Y, Huynh VN, Lawry J (eds) International Conference on Integrated Uncertainty in Knowledge Modeling and Decision Making, Berlin: Springer-Verlag, pp 7-15

Yang JB, Liu J, et al (2006) Belief rule-base inference methodology using the evidential reasoning approach - RIMER. IEEE T Syst Man Cy A 36(2):266-285 